\title{
Prior probability modulates anticipatory activity in category-specific areas
}

\author{
Sabrina Trapp ${ }^{1}$ Jöran Lepsien ${ }^{1} \cdot$ Sonja A. Kotz ${ }^{1,2} \cdot$ Moshe Bar $^{3}$
}

Published online: 8 September 2015

(C) Psychonomic Society, Inc. 2015

\begin{abstract}
Bayesian models are currently a dominant framework for describing human information processing. However, it is not clear yet how major tenets of this framework can be translated to brain processes. In this study, we addressed the neural underpinning of prior probability and its effect on anticipatory activity in category-specific areas. Before fMRI scanning, participants were trained in two behavioral sessions to learn the prior probability and correct order of visual events within a sequence. The events of each sequence included two different presentations of a geometric shape and one picture of either a house or a face, which appeared with either a high or a low likelihood. Each sequence was preceded by a cue that gave participants probabilistic information about which items to expect next. This allowed examining cue-related anticipatory modulation of activity as a function of prior probability in category-specific areas (fusiform face area and parahippocampal place area). Our findings show that activity in the fusiform face area was higher when faces had a higher prior probability. The finding of a difference between levels of expectations is consistent with graded, probabilistically modulated activity, but the data do not rule out the alternative explanation of a categorical neural response. Importantly, these differences were only visible during anticipation, and vanished at the time of stimulus presentation, calling for a
\end{abstract}

Sabrina Trapp

strapp@cbs.mpg.de

1 Max Planck Institute for Human Cognitive and Brain Sciences, Stephanstrasse 1a, D-04103 Leipzig, Germany

2 School of Psychological Sciences, The University of Manchester, Manchester, England

3 Gonda Center for Brain Research, Bar-Ilan University, Ramat Gan, Israel functional distinction when considering the effects of prior probability. Finally, there were no anticipatory effects for houses in the parahippocampal place area, suggesting sensitivity to stimulus material when looking at effects of prediction.

Keywords Anticipation · Predictive coding · Fusiform face area $\cdot$ Bayesian $\cdot$ Statistical learning $\cdot$ Likelihood

\section{Introduction}

Being able to estimate and take into account the certainty of evidence is important for optimal decision-making and behavior. In the past decade, Bayesian models have predominantly been used to describe and explain a vast amount of behavioral (e.g., Ernst \& Banks, 2002; Körding \& Wolpert, 2004; Tassinari, Hudson, \& Landy, 2006) and imaging (e.g., Vilares, Howard, Fernandes, Gottfried \& Kording, 2012) data pertaining to the role of probability in perception and action. In Bayesian models, two sources of evidence are relevant in order to infer the posterior probability of sensory data: prior probability and likelihood. For example, to decide whether the sensory data are consistent with a white rabbit in a befogged top hat, both the prior probability of a white rabbit appearing in a top hat and the current sensory evidence, the likelihood, have to be taken into account. The basic premise of such models is that the brain represents information probabilistically (Knill \& Pouget, 2004). At the level of large-scale networks, modulations as a function of probability of occurrence have been investigated in a number of reinforcement learning studies, where a reward is delivered only with certain likelihood. For example, Abler et al. (2006) used a simple delayed incentive task with a discrete range of reward probabilities. During the anticipation period of the task, activation in the 
nucleus accumbens increased linearly with the probability of a given reward. Similarly, Knutson et al. (2005) found that activity of the mesial prefrontal cortex increased proportionally to gain probability. However, brain areas identified with reinforcement learning may rather mediate processes such as stimulus-independent value expectations. To probe for neural correlates of stimulus-specific prior probabilities, the task has to tap into more perceptual processes. In a study by Vilares et al. (2012), participants had to estimate the position of a hidden visual dot. At the beginning of each trial, they received information about its likely position in the form of surrounding dots, manipulating its likelihood. In addition, participants could obtain the prior probability of the target position from the distribution of previous target positions. The authors found that likelihood modulated higher-level sensory regions (bilateral regions of superior occipital visual cortex), while prior probability modulated activity in the putamen, amygdala, insula, and orbitofrontal cortex. The regions they identified as related to prior probability are commonly not related to specifically represent stimulus information. Of note is that while the position of the target could change, its identity always remained the same (i.e., a dot). It remains possible that prior probability directly influences activity in regions where the actual stimulus is represented by increasing overall baseline activity. Such modulation could serve as a "neural weight" of prior probability, and contribute to the computation of the posterior probability in sensory-specific regions. To investigate this option, one would have to manipulate prior probability of stimulus information and probe for subsequent modulations in stimulus-specific regions. In a study by Summerfield and Koechlin (2008), participants made perceptual decisions and were either biased in favor of a single visual alternative or had no prior expectation. The authors reported increased activity in extrastriate regions in the bias condition, indicating the pre-activation of the representation of the stimulus. However, the prior probability was not manipulated in this study; rather, participants were either biased in favor of one alternative or not.

We aimed to examine whether prior probabilities directly modulate sensory activity during anticipation. Specifically, we asked whether the BOLD signal can be modulated as a function of prior probability in brain regions that are assumed to represent category stimulus information. There is a lot of evidence that the fusiform face area (FFA) is specialized for processing faces (Kanwisher, McDermott, \& Chun, 1997) and the parahippocampal place area (PPA) for processing scenes and buildings (Epstein \& Kanwisher, 1998). Not only have these areas been shown to be sensitive to the respective categories but both imagery and expectation of faces and houses lead to activity increases in FFA and PPA, respectively (Esterman \& Yantis, 2009; Ishai, Ungerleider, \& Haxby, 2000; O'Craven \& Kanwisher, 2000; Puri, Wojciulik \& Ranganath, 2009). Egner et al. (2010) capitalized upon those findings and used a task in which a cue was followed by face stimuli that occurred with a high, medium, or low probability. The authors examined stimulus-related activity in the FFA as a function of face expectation (face was expected and shown), surprise (face was expected but house was shown), and the interaction between expectation and surprise. The authors found a significant interaction: Under high face expectation, there was no difference in FFA activity when faces or houses were shown. Interestingly, they found no main effect of expectation in FFA, i.e., a modulation as a function of prior probability. Taken together, these data do not exclude the interpretation that anticipation was binary, i.e., an expectation of faces in the high, but not in the low and medium condition. A possible explanation is that the response at stimulation obscures differences between prior probabilities. While it is possible that less neural resources are allocated to events with a low prior probability during anticipation, such sensory events may elicit a higher prediction error at stimulation, which has been shown to increase the BOLD signal, for example, in FFA (de Gardelle, Waczuzk, Egner, \& Summerfield, 2013; Egner, Monti, \& Summerfield, 2010). Conversely, events with a high prior probability might elicit a higher baseline shift during anticipation, but a lower BOLD response at stimulation, due to a lower prediction error. Therefore, a second goal of this study was to directly distinguish between anticipation and stimulation, and address whether prior probability modulates anticipatory BOLD response, i.e., before stimulus onset. We hypothesized that we would find a higher anticipatory BOLD response if the faces had a high prior probability. The same pattern was expected for PPA and house stimuli. In addition, in a more exploratory fashion, we probed for temporal distance effects, i.e., whether an anticipatory BOLD signal would be stronger if a stimulus occurred earlier rather than later in time.

\section{Material and methods}

\section{Participants}

Twenty volunteers were recruited from the Max Planck Institute for Human Cognitive and Brain Science community and provided written informed consent. They were financially compensated for their participation. All participants had normal or corrected-to-normal vision. The study was carried out according to the guidelines approved by the Ethics Committee of the University of Leipzig. One participant was not successful in the memory test of the behavioral training session, and another participant was not able to attend the functional magnetic resonance imaging (fMRI) sessions. We therefore excluded both from the study sample. The remaining 18 volunteers participated in four sessions, each lasting approximately 1 hour (see "Procedure" section). For two more participants, 
behavioral data from one of two fMRI sessions were either not recorded or incomplete (less than $10 \%$ responses). The analyses were performed with the remaining 16 participants.

\section{Stimuli}

Ten different visual sequences were used in the present study. Each sequence consisted of a geometric pattern, a textured rectangular, and either a picture of a face or a house, with the order of presentation varying between conditions (see below).

Each sequence was preceded by a cue, which was one of the letters A, B, C, D, or E. Each of these five cues was associated with two different sequences; one of the sequences contained a picture of a face, and the other one contained a picture of a house. Altogether, the set consisted of ten different sequences. The cues differed in the probability with which they were associated with a face or a house picture, either with a high (70\%) or a low (30\%) probability (see Fig. 1). In addition, the sequences differed in the position of the face and the house (either on position 1 or position 3). The two sequences associated with the letter $\mathrm{E}$ were introduced as a baseline for comparison. They contained neither a picture of a face nor a house, but a picture of a globe $(70 \%)$ and a picture of a bag (30\%). The cue allowed participants to anticipate the complete sequence (see "Procedure" section).

Note that we excluded potential effects of familiarity and, thereby, differences as a function of memory retrieval for each sequence type by re-using the same stimuli/sequence for both face [house] low and high probability conditions (A and C, and $\mathrm{B}$ and $\mathrm{D}$, respectively). Thereby, we avoided anticipatory BOLD modulation from being contaminated by factors other than prior probability or temporal distance.

\section{Design}

Figure 1 shows the design of the study.

\section{Procedure}

Training sessions before the scanning experiment There were two behavioral training sessions, separated by 3 days. In the first training session, participants were shown all ten sequences and their associated cues (see "Stimuli", Fig. 1) on a piece of paper and were given $15 \mathrm{~min}$ to memorize them. They were instructed that they will later view these sequences on a PC and that they will have to detect an order deviant, i.e., a change in the memorized position of the picture within the sequence.

After the initial acquaintance with and memorization of the stimulus material, participants started a computer-based training, during which they were exposed to sequences. Some of these sequences were shown with high probability ( $70 \%$ of all trials) and some sequences were shown with low probability (30\% of all trials). The exact trial structure was as follows: One of the five possible cues (A-E) was presented on the screen for duration of $1,000 \mathrm{~ms}$. After a variable delay ( 1 , $500,2,000$, and 2,500 ms), three pictures were presented consecutively, each for $250 \mathrm{~ms}$, with an inter-stimulus interval of $250 \mathrm{~ms}$. The task structure is illustrated in Fig. 2. ${ }^{1}$ The rationale was that participants would store the associative strength between cue and visual events after several exposures, and, in addition, that they would anticipate the sequence order with cue presentation to be better prepared to detect a deviant that occurred in $10 \%$ of all trials. No feedback was given. The sequence task lasted $32.2 \mathrm{~min}$ per session.

After 3 days, participants returned for a second training session. At the beginning of this session, they were given the sequence events (cue plus pictures) on single cards and were asked to put them in the right order and to indicate which sequence occurred more often (high vs. low). It was important that participants were aware of probabilistic contingencies to avoid variance due to implicit versus explicit formation of expectations. Only if participants were successful (i.e., no error) in this memory evaluation did they start with the second PC training, which was identical to the training of session 1. For the training sessions, we did not emphasize response speed because we primarily wanted to ensure that participants learned the correct order of each sequence and internalized different probability distributions. In contrast, we emphasized speed during the fMRI sessions to encourage them to use the cue to anticipate the sequence to be readily prepared for the quick detection of an order deviant. Participants were informed after the second training session that speed was to be emphasized during the scanning session.

The training sessions were presented on a 19-in computer screen with the Presentation software version 14.7 (Neurobehavioral Systems, Inc., Berkley, CA, USA).

fMRI session Two days after the second behavioral training, participants returned for the fMRI session. The scanning session consisted of three parts: memory test, sequence task, and a localizer scan to identify regions related to processing faces (FFA) and houses (PPA).

At the beginning, participants had to perform the memory test again. Only upon successful completion of the memory test (i.e., no errors) did participants continue with the scanning procedure. If participants had just one error, he/she was excluded from further testing. Following this procedure, one participant was excluded (see "Participants" section).

\footnotetext{
${ }^{1}$ Note that there was a difference in the delay between cue and sequences for the behavioral training $(1,500,2,000,2,500 \mathrm{~ms})$ and the fMRI session $(6,000,7,000,8,000 \mathrm{~ms})$ to be able to probe for an event-related BOLD response in the latter.
} 
Faces high probability, short distance
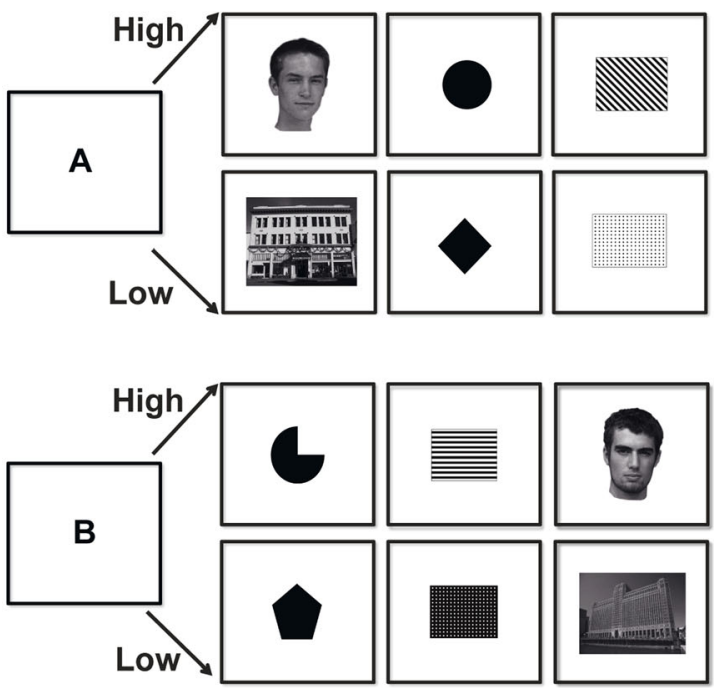

Faces high probability, long distance

Fig. 1 Stimuli and conditions used in the experiment. Participants learned visual sequences with different pictures. The pictures were presented serially, starting with a cue (letter). Each letter cue was associated with two sequences: (a) a sequence with high face [house]

Participants then proceeded with the task, which was identical to the training session, except that the ISI, i.e., the delay between the cue and the first sequence event, was longer (6, $000,7,000,8,000 \mathrm{~ms})$. We chose a longer delay period to make sure participants would have sufficient time to anticipate the subsequent sequence, and to be able to analyze the fMRI data in an event-related fashion. It is important to note that we deliberately chose an unpredictable onset of stimulation to prevent participants anticipating the temporal onset of the first picture in the sequence, thereby confounding the BOLD response with temporal expectation. Response speed was emphasized to encourage participants to anticipate the sequence for a quick detection of an order deviant. The deviants were randomly distributed across the experiment (10\%). Therefore, it was not possible to anticipate whether the subsequent sequence would contain an order deviant or not. Accordingly, the exact button press could
Faces low probability, short distance
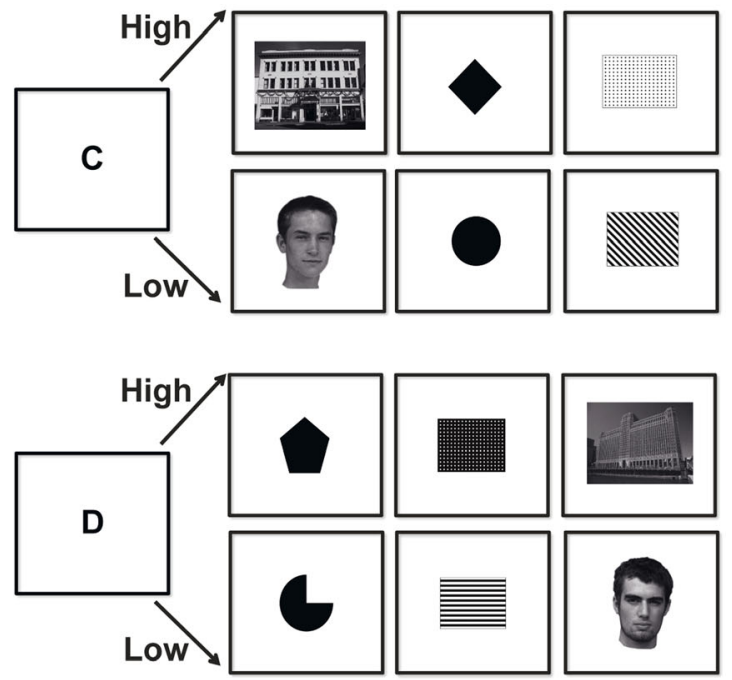

Faces low probability, long distance

prior probability [70\%] and (b) a sequence with low face [house] prior probability [30\%]. The face [house] picture was presented either on position 1 (short temporal distance) or position 3 of the sequence (long temporal distance)

not be anticipated, thus preventing BOLD activity to be confounded with the anticipatory specification of a motor response.

For the subsequent localizer task, participants viewed serially presented pictures of faces, outdoor scenes, or checkerboard-like grid stimuli, and indicated with a speeded choice response whether the presented stimulus matched the previous one (continuous 1-back task). In each block, 11 stimuli from one category were presented for $1 \mathrm{~s}$, separated by a 1s inter-stimulus-interval (block length: $22 \mathrm{~s}$ ). Within each block, there were five matches and five non-matches. There were six blocks for each category (faces, scenes, checkerboards), separated by 6-s inter-block intervals. The order of stimuli within each block and the order of blocks were pseudo-randomized, with the exception of every fourth block, which was a 22-s baseline block without stimulation. The localizer lasted $10.4 \mathrm{~min}$, and was conducted in each session.

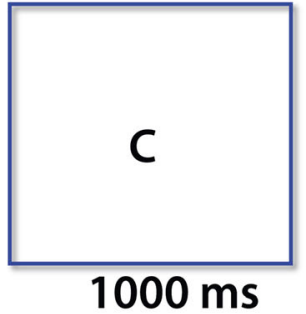

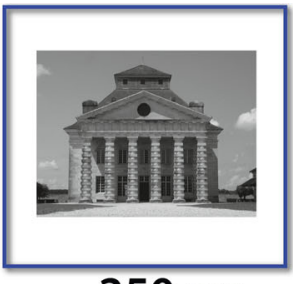

$250 \mathrm{~ms}$

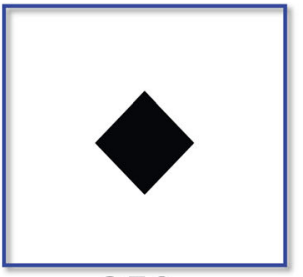

$250 \mathrm{~ms}$

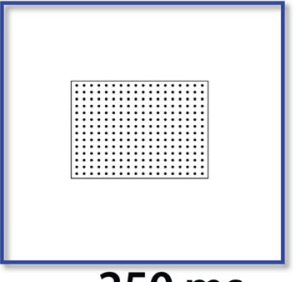

$250 \mathrm{~ms}$

$6,7,8 \mathrm{~s}$ $250 \mathrm{~ms}$ $250 \mathrm{~ms}$

Fig. 2 Order deviant detection task. Participants had to detect an order deviant within the sequence, i.e., a picture presented at an incorrect position. The correct order of pictures in the sequence was learnt beforehand. They had to react as fast as possible to a deviant or to no deviant, i.e., correct order. A deviant occurred in $10 \%$ of all trials. The inter-stimulus interval was jittered between 6,000, 7,000, and 8,000 ms 
During the fMRI session, participants lay inside the scanner and viewed the stimuli through a mirror projected onto a back-projection screen by an LCD projector. Stimulus presentation and behavioral response collection were controlled by Presentation software (Neurobehavioral Systems, Inc.). The fMRI session was split into two consecutive sessions in order to keep each session at an endurable length.

fMRI data recording and analysis Images were collected with a 3 T Magnetom TimTrio MRI scanner system (Siemens Medical Systems, Erlangen, Germany) using a 12channel head coil. Thirty axial slices were collected parallel to the AC-PC plane using a gradient-echo EPI sequence with a TE $28 \mathrm{~ms}$, flip angle $90^{\circ}$, TR 2,000 ms, acquisition bandwidth $2232 \mathrm{~Hz} / \mathrm{px}$, with $3 \mathrm{~mm}^{3}$ and inter-slice gap of $1 \mathrm{~mm}$. The matrix acquired was $64 \times 64$ with a field of view (FOV) of $19.2 \mathrm{~cm}$. The experiment was split into two sessions, each consisting of 971 EPI volumes. High-resolution T1-weighted MR scans of the participants were acquired beforehand and were part of the Max Planck Institute for Human Cognitive and Brain Science database.

fMRI data were analyzed using Statistical Parametric Mapping (SPM 12b; WellcomeTrust Centre for Neuroimaging, UCL). Data were preprocessed including correcting for differences in slice acquisition as well as for motion and image distortions (based on separately acquired fieldmaps of the local static magnetic field). Functional data were normalized to the Montreal Neurological Institute template, using the unified segmentation approach (Ashburner \& Friston, 2005) and spatially smoothed with a Gaussian kernel of 8-mm full width at half maximum (FWHM). In addition, a high-pass filter was applied using cutoff frequency of $1 / 128 \mathrm{~Hz}$. The intra-individual statistical analysis was based on a least-squares estimation using the general linear model taking the non-sphericity into account.

The model of the sequence task included regressors coding for the onsets and durations of the following phases of each trial: cue, delay, pictures, and response. The focus of the present study was the delay period following the cue before onset of the sequence, that is, the period when participants were able to anticipate an upcoming sequence. To examine activity as a function of prior probability and temporal distance of the categoryspecific stimuli, the delay period was separated into five different regressors, each coding for one of five possible cues (A, B, C, D, and cue E). Cue-condition E (only containing pictures of a globe and a bag) served as a high-level baseline, allowing controlling for global effects unspecific to category-anticipation effects in FFA and PPA. Therefore, this cue was subtracted from others cues, and the contrast estimates of the remaining regressors were later analyzed under a $2 \times 2$ design (prior probability [high,low], temporal distance [short,long]) in the region-of-interest (ROI) analysis.

The localizer task was analyzed in a comparable fashion, but implementing a block design and using a high-pass filter of 1/180 s. Face-sensitive areas were identified by contrasting faces versus scenes using a liberal threshold of $p<0.001$ (uncorrected). A corresponding analysis was done for scenesensitive areas, where we contrasted scenes versus faces. If for a participant it was not possible to find clear activations in these ROIs, the threshold was lowered. If still no maximum was identifiable, the mean coordinates derived from all other participants were used. For 12 participants, one or more out of four coordinates (left and right FFA and PPA, respectively) could only be identified with lowered thresholds. For three participants, coordinates were partially replaced by the group mean (right FFA: 42/-50/-18, left FFA: -39/-51/-18, right PPA: 28/-46/-9, left PPA: -27/-48/-10). We used these ROIs (size: one voxel) to assess the difference between the experimental conditions. All ROI analyses were performed using the rfxplot toolbox (Gläscher, 2009). The resulting contrast estimates were analyzed with an ANOVA using SPSS.

\section{Results}

\section{Behavioral}

The accuracy was at ceiling in both sessions; therefore, we did not subject the data to inferential statistics. In fMRI session 1, average performance was $95.2 \%$ correct $(\mathrm{SD}=6.31 \%)$ for sequences with a high prior probability, and $95.4 \%$ correct on average ( $\mathrm{SD}=5.52 \%$ ) for sequences with a low prior probability. Likewise, during the fMRI session 2, overall performance was at ceiling both for sequences with a high $(92.3 \%$ correct on average, $\mathrm{SD}=11.8 \%)$ and low $(93.4 \%$ correct on average, $\mathrm{SD}=10.63 \%$ ) prior probability.

Reaction times (RTs) were fast on average (fMRI session 1: high probability: $230 \mathrm{~ms}$; SD: $44.3 \mathrm{~ms}$, low probability: $242.2 \mathrm{~ms}$; SD: $46.4 \mathrm{~ms}$; fMRI session 2: high probability: $251.2 \mathrm{~ms}$; SD: $36.3 \mathrm{~ms}$; low probability: $251 \mathrm{~ms}$; SD: $34.1 \mathrm{~ms}$ ) and did not differ between high and low probability conditions $(\mathrm{t}(15)=0.016, p=0.987)$.

Note that the study was not designed as a RT experiment. The rationale for our study was to teach associative strengths between cues and sensory events by mere exposure, ${ }^{2}$ and the order deviant task was conceptualized as a cover-task towards this end. We assumed that independent from the functional requirement of the task, the prior probabilities of stimuli

\footnotetext{
${ }^{2}$ Although participants were eventually informed that there were different frequencies of specific sequences (by asking them which sequence occurs more often in the second training session), they were not informed about the exact amount of exposure (70 \% vs. $30 \%)$.
} 
would nevertheless be learned, stored, and retrieved according to their likelihood eventually. Due to this emphasis in the design, the analysis of RT data suffers from technical constraints. The picture presentation and the subsequent interstimulus interval (ISI) were both very short (each $250 \mathrm{~ms}$ ), leaving little time to react before the onset of the next stimulus. Presentation software logs the response to the current event, and therefore, it may be that a (too late) response to picture 1 [2] is logged to the onset of the subsequent picture (i.e., picture 2 [3]), rendering the response latency artificially low. In principle, this problem could be solved by analyzing only those remaining trials in which participants responded to the correct picture position. However, the problem is that although speed was emphasized, some participants already made their decision about the correctness of the order with picture 2 (as here the third position is already determined) whereas other (more uncertain) participants only made their button press once the last picture 3 was shown. Therefore, RT analysis suffers from certain limitations.

\section{Whole-brain analysis}

The focus of the present study was to examine modulation of activity in two category-specific areas as a function of prior probability. It is important to note that the only difference between the experimental conditions was the prior probability (high vs. low) and the temporal distance of faces and houses in the sequence (early vs. late) while stimuli and task were identical across conditions. Therefore, a wholebrain analysis would necessarily result only in activation differences in regions that represent and process prior probabilities or temporal onset of upcoming sensory events. However, the whole-brain analysis showed no significant clusters that survived family-wise error correction for the difference between cue A and cue C (high [low] vs. low [high] prior probability of faces [houses]), cue $\mathrm{A}$ and cue $\mathrm{B}$ (short vs. long temporal distance of faces), and cue $\mathrm{C}$ and cue D (short vs. long temporal distance of houses). As the location of FFA and PPA shows significant variation between participants, we here focused on a ROI analysis in these regions to probe for differences as a function of prior probability and temporal distance, based on individually determined coordinates extracted from the localizer task.

\section{Region-of-interest analysis}

There was a stronger signal during anticipation in the FFA if faces had a high as compared to a low prior probability (left: $\mathrm{F}(1,15)=6.8, p=0.020$; right: $\mathrm{F}(1,15)=7.42, p=0.016$; ANOVA repeated-measures). There was no main effect of temporal distance (left: $\mathrm{F}(1,15)=0.2, p=0.6665$; right: $\mathrm{F}(1,15)=0.19, p=0.669)$. The interaction between prior probability and temporal distance was significant for the left
$(\mathrm{F}(1,15)=9.83, p=0.007)$ but not for the right $\mathrm{FFA}(\mathrm{F}(1,15)=$ $0.71, p=0.412$ ). We found no evidence for any modulations in PPA for house stimuli as a function of prior probability (left: $\mathrm{F}(1,15)=0.8, p=0.387$; right: $\mathrm{F}(1,15)=3.92, p=0.067)$ or temporal distance (left: $\mathrm{F}(1,15)=1.41, p=0.253$; right: $\mathrm{F}(1,15)=0.544, p=0.472)$. For illustration of the results see Fig. 3.

Note that to truly reflect sensitivity to prior probability, a neural correlate should show activation that parametrically varies with the degree of expectation. Therefore, in a second step, we explicitly compared activity related to cue E (no face expectation) to low face expectation (cue $\mathrm{C}$ ) and high face expectation (cue A). In accordance with the $2 \times 2$ ANOVA model, paired t-tests revealed a significant difference in FFA between high versus low prior probability of faces (cue A vs. C; right: $\mathrm{t}(15)=2.7, p=0.018$; left: $\mathrm{t}(15)=3.3, p=0.005)$ and between high prior probability and no face expectation (cue A vs. E; right: $\mathrm{t}(15)=2.3, p=0.040$; left: $\mathrm{t}(15)=2.5, p=0.022$ ). However, there was no statistically significant difference between low prior probability and no face expectation (cue $\mathrm{C}$ vs. $\mathrm{E}$; right: $\mathrm{t}(15)=0.1, p=0.916$; left: $\mathrm{t}(15)=0.03, p=0.974)$.

For the PPA, we again found no significant difference between cues associated with high versus low prior probability of houses in right and left PPA (cue A vs. C; right: $\mathrm{t}(15)=-1.6$, $p=0.125$; left: $\mathrm{t}(15)=-0.3, p=0.779)$. There was also no significant difference between high prior probability and no house expectation (cue $\mathrm{C}$ vs. E; right: $\mathrm{t}(15)=-0.5, p=0.602$; left: $\mathrm{t}(15)=-1.4, p=0.190)$, and low prior probability and no house expectation (cue A vs. E; right: $\mathrm{t}(15)=-1.4, p=0.171$; left: $\mathrm{t}(15)=-01.6, p=0.141)$.

Analogous to previous studies, we also looked at activity in FFA and PPA at the time of visual stimulation as a function of prior probability of stimuli. To this end, we set up an additional model including ten different regressors that coded for the onset of the sequence as a function of the prior probability of the picture and the actual picture shown in this trial. For example, for faces, when cue A was shown, there were two different regressors for the onset of this sequence: (a) a regressor with a high prior probability of faces, and a face shown at presentation, and (b) a regressor with a high prior probability of faces, and a face not shown at presentation. We restrained our analysis to those two sequences where the first picture was a face or a house (i.e., cue A and C), because all the following events were confounded with participants' responses, as the task was primarily designed to analyze the anticipation period. We then probed for differences in right and left FFA [PPA] when a face [house] was presented and had a high prior probability (cue $A[C\}$ ) versus when a face was presented and had a low prior probability (cue $\mathrm{C}[\mathrm{A}\}$ ). The difference in the FFA was not significant (right: $\mathrm{t}(15)=.688, p=0.502$; left: $\mathrm{t}(15)=0.33, p=0.974)$. The data also did not show a significant difference in the PPA (right: $\mathrm{t}(15)=-.050, p=0.961$; left: $\mathrm{t}(15)=-.068, p=0.947)$. 

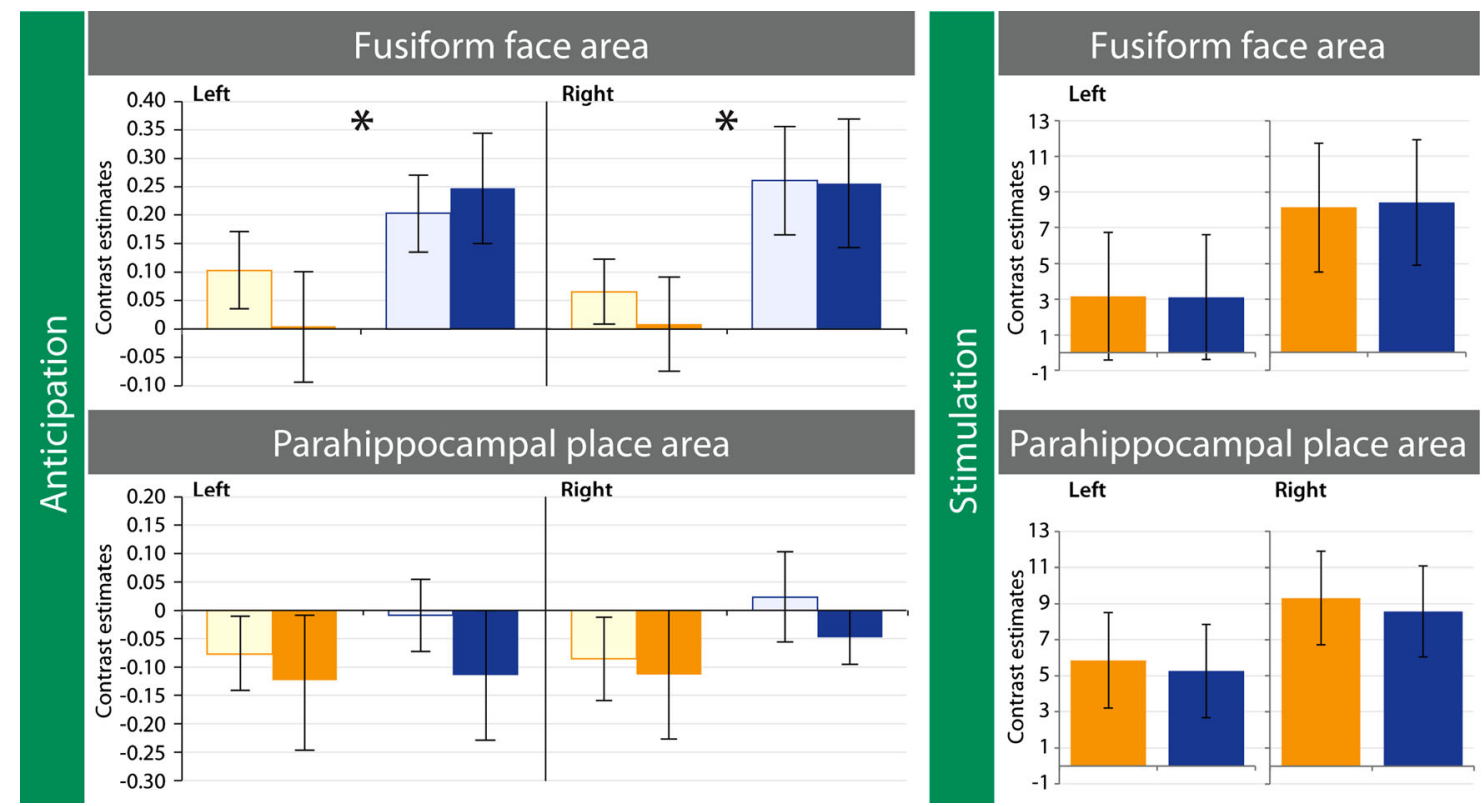

Fig. 3 Region-of-interest analysis. Contrast estimates as a function of prior probability and temporal distance in fusiform face area (top) and parahippocampal place area (bottom), during anticipation (left) and stimulation (right). The color code used is: dark blue $=$ high prior

\section{Discussion}

A wealth of studies leave little doubt that expectations have an effect on stimulus-evoked activity (e.g., Alink et al., 2010; Den Ouden et al., 2009; Kok et al., 2012a, 2013; Todorovic et al., 2011). However, the question of whether this activity is modulated by prior probability during anticipation remained open. We designed a study that aimed to fill this gap and found that activity in FFA, a region specialized for face processing, was modulated by the prior probability of faces during anticipation. While we found a significant difference between high and low prior probability, a comparison between low prior probability (cue C) and no face expectation (cue E) revealed no difference. These results could in principle indicate that the difference between high and low prior probabilities is driven by a binary effect of expectation, that is, only anticipation of the highly likely sequence and its associated visual events. However, it has to be noted that the no face expectation condition, i.e., activity related to cue $\mathrm{E}$, was not ideally suited as a proper baseline. First, it is known that FFA is not only activated by faces, but can be modulated by perceptual expertise with, for instance, cars and birds (Gauthier et al., 2002) or novel 3D objects (Gauthier and Tarr, 2002; Wong et al., 2009). Participants in the current study were exposed to the two pictures that were associated with letter cue E over an extended period of four sessions. It is therefore possible that this led to some sort of perceptual expertise and, accordingly, to elevated activity in FFA, blurring differences with the low prior probability condition. Furthermore, errors in both conditions (high and low prior probability) did not differ and were probability, short temporal distance; light blue = high prior probability, long temporal distance; dark orange $=$ low prior probability, short temporal distance; light orange $=$ low prior probability, long temporal distance

low, supporting the notion that participants did prepare for both events. Certainly, low error rates could in principle also indicate that the task was easy and did not require any preparation. However, if this was true, one would either expect (a) a general, non-specific face expectation for all cues associated with faces - in this case, activity linked to cues with low prior probability should be different from cues linked to no face expectation, or (b) no face expectation at all - in this case, one would not expect a difference between high prior probability and no face expectation. Furthermore, an informal posttask report collected from the participants revealed a tendency to anticipate both the high and low likely sequence during the extended delay period. Altogether, this renders the option that expectation was entirely binary unlikely.

We did not find modulation of activity by prior probability of houses in the PPA. Similarly, Summerfield et al. (2006) did not find evidence for top-down modulation of PPA when they investigated how category-specific visual regions respond during erroneous perceptual judgments. In their study, participants had to discriminate between degraded, noisy pictures of houses and faces. The FFA was activated when a house was misperceived as a face, but the PPA was not activated when faces were misperceived as houses. The authors argued that the FFA might be strongly modulated by a top-down expectation that a face will be presented, explaining input as corresponding to a face stimulus even if a face stimulus was not present. While such a top-down expectation of faces might be facilitated by the overall, highly predictable structure of a face, natural scenes as well as buildings/houses entail larger variability, possibly making them less suited candidates for 
predictive processing. However, in our design, there were only two house pictures, rendering such an explanation less likely. An alternative explanation could be linked to the fact that the PPA is activated by mere associations (Aminoff et al., 2007; Bar et al., 2008). It is possible that the long ISIs during anticipation afforded general associative processes, which lead to PPA activation, and this in turn might have rendered the difference among high and low prior probability in PPA insignificant. Along these lines, PPA activity could have also been elevated by no house expectation (cue E), simply by associating the visual events of the sequence (globe and the bag picture) with the cue, or with each other. Finally, there is evidence that the "default network" overlaps with the network activated by contextual associations, which are assumed to provide the building blocks for predictions (Bar, 2007). Thus, the long ITIs serving as an implicit baseline in our design might have been confounded with PPA activity as well.

Vossel, Thiel, and Fink (2006) indirectly tested the influence of prior probability by manipulating cue validity (high vs. low), and probed for neural activation patterns in a visual target detection task. Data revealed higher activation of right inferior parietal and right frontal cortex for high compared with low cue validity. However, both parietal and frontal areas have been linked to the "source" of attentional processing (Corbetta et al., 1998). Generally, sources refer to brain systems that send top-down signals to sensory areas, whereas "sites" are assumed to be those target regions that are influenced by these top-down signals (Kastner \& Ungerleider, 2000; Saalmann et al., 2007). A few studies have directly investigated anticipatory activity in such site regions. Puri et al. (2009) reported that baseline activity in FFA was higher during expectation of a face versus expectation of a house and counter-likewise for PPA, indicating that these areas are generally sensitive to expectation. However, the important difference from our study is that these authors looked at FFA activity when either houses or faces were expected, whereas we examined modulations in this region when only faces were expected (with a high vs. a low prior probability). In a similarly relevant study by Esterman and Yantis (2009), participants had to detect a face or a house that gradually emerged from noise, and were cued to either faces or houses. The authors found better performance and higher baseline shifts in FFA and PPA when participants were biased towards a specific category. However, their cue was always $100 \%$ valid, i.e., activity was compared between conditions in which participants were either biased towards a category or not at all. Vilares et al. (2012) directly investigated the question of differential activation of the BOLD signal as a function of prior probability. However, the regions identified in that study, such as the putamen, the amygdala, the insula, and the orbitofrontal cortex, are all related to more general evaluation processes, and much less linked to the representation of an actual stimulus or category-specific information. Our motivation was to probe for modulations as a function of prior probabilities in regions that are assumed to actually represent and process sensory information. This comparison was not possible in the design of Vilares and colleagues (2012), as the authors compared conditions in which the identity of sensory information did not change, just its spatial position - the target's identity was always a dot. Although they did find modulation of higher-level visual areas as a function of likelihood, we here were primarily interested in whether such regions would also be modulated as a function of prior probability. Egner et al. (2010) manipulated the prior probability that a face will be shown (low, medium, high), and examined stimulus-related activity when a face was shown (main effect of expectation), a house was shown (main effect of surprise), and the interaction hereof. They found that in conditions with a high face expectation, but with a house stimulus shown, the activity in FFA was akin to the activity when a face was actually shown. This, however, could also mean that subjects had an expectation of a face in the high face expectation (vs. having no expectation of a face in the low and medium condition), which was responsible for the increase in FFA activity. Such an interpretation would be in line with a study that demonstrated FFA activity when a house is misperceived as a face (Summerfield et al., 2006). Possibly, the authors would have found a main effect of expectation had they looked at neural responses during anticipation rather than at stimulation, because neural response at stimulation may be a mixture of two processes - computation of prior probability and prediction error. A high prior probability may enforce the allocation of more resources in advance, and an increase in the BOLD response. In contrast, a low prior probability may lead to less resources allocated in advance, but a higher prediction error, which however also increases the BOLD response. Indeed, we did not find a main effect of prior probability when analyzing activity at the time of stimulus presentation. In addition to demonstrating the influence of prior probabilities on anticipatory neural signals, our result calls for a careful distinction between anticipation and stimulation when investigating effects of expectation.

We also probed for differences in the anticipatory BOLD response as a function of temporal distance of stimuli in a series of upcoming visual events. We hypothesized a larger response for events that were about to occur earlier in time. In contrast to our expectation, we did not find that the anticipatory BOLD signal was modulated as a function of the temporal onset of faces in a sequence. It is possible that faces constitute a stimulus category with a strong social significance and if likely to occur, may therefore be anticipated independent of whether they are about to appear a few seconds earlier rather than later. Another explanation is related to the sluggishness and long latency of the BOLD signal. It is conceivable that early signals were stronger for faces that were about to appear at the beginning of the sequence, but that this 
difference was rapidly overshadowed by anticipation of faces occurring later in time. Future studies may address such questions with methods that offer better temporal resolution such as electroencephalography or magnetoenceohalography.

It should be noted that the processes that underlie these and related effects of preparatory activity can be interpreted with or linked to different psychological constructs. For example, Peelen and Kastner (2011) investigated anticipatory activity when preparing for a certain object category (people vs. cars) and related their findings to attention. In an fMRI study by Kok et al. (2014), an auditory cue predicted the orientation of a subsequently presented grating stimulus. The pattern of activity evoked with this cue was similar to that pattern evoked by the actual stimulus in primary visual cortex, and the authors interpreted their findings as reflecting expectations. Another construct that is presumably linked to anticipation is imagery. Stokes et al. (2009) asked participants to imagine either X or $\mathrm{O}$, and revealed with fMRI that the pattern of activation resembles that of actual visual stimulation. In the informal posttask debriefing, our participants also reported that that they "imagined" upcoming pictures. It is possible that predictions or expectations that entail conscious awareness may include active imagery and simulation of upcoming events. However, constructs such as "imagery" are generally too vague and unspecific to be discussed as alternative candidate mechanisms. Attention, on the other hand, is a construct that has been more specified and there is an ongoing debate about how attention and expectation are linked (Kok et al., 2012b; Summerfield \& Egner, 2009). A study utilizing signal detection analysis suggests that attention affects the actual sensitivity for visual input, whereas expectation adjusts the response criterion (Wyart et al., 2012). Clearly, the current design does not allow disentangling attention to and expectation of upcoming information. We were primarily interested in the question of whether prior probability would modulate anticipatory activity in category-specific regions, and our results suggest that a probability-sensitive baseline shift in FFA might serve as a "neural bias" towards the more likely category interpretation. Our results leave open the question of which psychological process actually subserves this bias. Differentiating among these constructs is certainly an important venue for future research, as the Bayesian framework is mute to the psychological processes that underlie the computation of and comparison between prior, likelihood, and posterior probabilities (Jones \& Love, 2011).

Acknowledgments This study was supported by a Max Planck fellowship (Dr. med. Anneliese \& DSc Dieter Pontius Foundation) (ST), DFG KO 2268/6-1(SK), and the Israeli Center of Research Excellence in Cognition (ICORE) grant 51/11 (MB).

\section{References}

Abler, B., Walter, H., Erk, S., Kammerer, H., \& Spitzer, M. (2006). Prediction error as a linear function of reward probability is coded in human nucleus accumbens. NeuroImage, 31, 790-795.

Alink, A., Schwiedrzik, C. M., Kohler, A., Singer, W., \& Muckli, L. (2010). Stimulus predictability reduces responses in primary visual cortex. Journal of Neuroscience, 30, 2960-2966.

Aminoff, E., Gronau, N., \& Bar, M. (2007). The parahippocampal cortex mediates spatial and non-spatial associations. Cerebral Cortex, 27, $1493-1503$.

Ashburner, J., \& Friston, K. J. (2005). Unified segmentation. NeuroImage, 26, 839-851.

Bar, M. (2007). The proactive brain: Using analogies and associations to generate predictions. Trends in Cognitive Sciences, 11(7), 280-289.

Bar, M., Aminoff, E., \& Schacter, D. (2008). Scenes unseen: The parahippocampal cortex subserves contextual associations, not scenes per se. Journal of Neuroscience, 28, 8539-8544.

Corbetta, M., Akbudak, E., Conturo, T. E., Drury, H. A., Linenweber, M., Ollinger, J. M., . . . Shulman, G. L. (1998). A common network of functional areas for attention and eye movements. Neuron, 21, 761773.

de Gardelle, V., Waczuzk, M., Egner, T., \& Summerfield, C. (2013). Concurrent representations of prediction and prediction error signals in visual cortex. Cerebral Cortex, 23(9), 2235-2244.

den Ouden, H. E., Friston, K. J., Daw, N. D., McIntosh, A. R., \& Stephan, K. E. (2009). A dual role for prediction error in associative learning. Cerebral Cortex, 19(5), 1175-1185. doi:10.1093/cercor/bhn161

Egner, T., Monti, J. M., \& Summerfield, C. (2010). Expectation and surprise determine neural population responses in the ventral visual stream. Journal of Neuroscience, 8, 16601-16608.

Epstein, R., \& Kanwisher, N. (1998). A cortical representation of the local visual environment. Nature, 392(6676), 598-601.

Ernst, M. O., \& Banks, M. S. (2002). Humans integrate visual and haptic information in a statistically optimal fashion. Nature, 415, 429-433.

Esterman, M., \& Yantis, S. (2009). Perceptual expectation evokes category-selective cortical activity. Cerebral Cortex, 20, 12451253.

Gauthier, I., Skudlarski, P., Gore, J. C., \& Anderson, A. W. (2002). Expertise for cars and birds recruits brain areas involved in face recognition. Nature Neuroscience, 3(2), 191-197.

Gauthier, I., \& Tarr, M. J. (2002). Unraveling mechanisms for expert object recognition: Bridging brain activity and behavior. Journal of Experimental Psychology Human, 28(2), 431-446.

Glascher, J. (2009). Visualization of group inference data in functional neuroimaging. Neuroinformatics, 7, 73-82.

Ishai, A., Ungerleider, L. G., \& Haxby, J. V. (2000). Distributed neural systems for the generation of visual images. Neuron, 28, 979-990.

Jones, M., \& Love, B. C. (2011). Bayesian Fundamentalism or Enlightenment? On the explanatory status and theoretical contributions of Bayesian models of cognition. The Behavioral and Brain Sciences, 34(4), 169-188. doi:10.1017/S0140525X10003134

Kanwisher, N., McDermott, J., \& Chun, M. M. (1997). The Fusiform Face Area: A Module in Human Extrastriate Cortex Specialized for the Perception of Faces. Journal of Neuroscience, 17, 43024311.

Kastner, S., \& Ungerleider, L. G. (2000). Mechanisms of visual attention in the human cortex. Annual Review Neuroscience, 23, 315-341.

Knill, D. C., \& Pouget, A. (2004). The Bayesian brain: The role of uncertainty in neural coding and computation. Trends in Neuroscience, 27(12), 712-719.

Knutson, B., Taylor, J., Kaufmann, M., Peterson, R., \& Glover, G. (2005). Distributed neural representation of expected value. Journal of Neuroscience, 25, 4806-4812. 
Kok, P., Failing, M., \& de Lange, F. P. (2014). Prior expectations evoke stimulus templates in the primary visual cortex. Journal of Cognitive Neuroscience, 26, 1546-1554

Kok, P., Brouwer, G. J., van Gerven, M. A., \& de Lange, F. P. (2013). Prior expectations bias sensory representations in visual cortex. Journal of Neuroscience, 33, 16275-16284.

Kok, P., Jehee, J. F. M., \& de Lange, F. P. (2012a). Less is more: Expectation sharpens representations in the primary visual cortex. Neuron, 75, 265-270.

Kok, P., Rahnev, D., Jehee, J. F. M., Lau, H. C., \& de Lange, F. P. (2012b). Attention reverses the effect of prediction in silencing sensory signals. Cerebral Cortex, 22, 2197-2206.

Kording, K. P., \& Wolpert, D. M. (2004). Bayesian integration in sensorimotor learning. Nature, 427, 244-247.

O'Craven, K. M., \& Kanwisher, N. (2000). Mental imagery of faces and places activates corresponding stimulus-specific brain regions. Journal of Cognitive Neuroscience, 12, 1013-1023.

Peelen, M. V., \& Kastner, S. (2011). A neural basis for real-world visual search in human occipitotemporal cortex. Proceedings of the National Academy of Sciences of the United States of America, 108(29), 12125-12130. doi:10.1073/pnas.1101042108

Puri, A. M., Wojciulik, E., \& Ranganath, C. (2009). Category expectation modulates baseline and stimulus-evoked activity in human inferotemporal cortex. Brain Research, 1301, 89-99.

Saalmann, Y. B., Pigarev, I. N., \& Vidyasagar, T. R. (2007). Neural mechanisms of visual attention: How top-down feedback highlights relevant locations. Science, 316, 1612-1615.

Stokes, M., Thompson, R., Cusack, R., \& Duncan, J. (2009). Top-down activation of shape-specific population codes in visual cortex during mental imagery. Journal of Neuroscience, 29(5), 1565-1572. doi: 10.1523/JNEUROSCI.4657-08.2009
Summerfield, C., \& Egner, T. (2009). Expectation (and attention) in visual cognition. Trends in Cognitive Sciences, 13(9), 403-409.

Summerfield, C., \& Koechlin, E. (2008). A neural representation of prior information during perceptual inference. Neuron, 59(2), 336-347.

Summerfield, C., Egner, T., Mangels, J., \& Hirsch, J. (2006). Mistaking a house for a face: Neural correlates of misperception in healthy humans. Cerebral Cortex, 16(4), 500-508.

Summerfield, C., \& Koechlin, E. (2008). A neural representation of prior information during perceptual inference. Neuron, 59(2), 336-347.

Tassinari, H., Hudson, T. E., \& Landy, M. S. (2006). Combining priors and noisy visual cues in a rapid pointing task. Journal of Neuroscience, 26, 10154-10163.

Todorovic, A., van Ede, F., Maris, E., \& de Lange, F. P. (2011). Prior expectation mediates neural adaptation to repeated sounds in the auditory cortex: An MEG study. Journal of Neuroscience, 31, 9118-9123.

Vilares, I., Howard, J. D., Fernandes, H. L., Gottfried, J. A., \& Kording, K. P. (2012). Differential representations of prior and likelihood uncertainty in the human brain. Current Biology, 22(18), 16411648.

Vossel, S., Thiel, C. M., \& Fink, G. R. (2006). Cue validity modulates the neural correlates of covert endogenous orienting of attention in parietal and frontal cortex. NeuroImage, 32, 1257-1264.

Wong, A. C. N., Palmeri, T. J., \& Gauthier, I. (2009). Conditions for facelike expertise with objects: Becoming a Ziggerin expert-but which type? Psychological Science, 20(9), 1108-1117.

Wyart, V., Nobre, A. C., \& Summerfield, C. (2012). Dissociable influences of signal probability and relevance on visual signal detection. Proceedings of the National Academy of Sciences of the United States of America, 109(9), 3593-3598. 